\title{
Prevalência de anticorpos anti-Toxoplasma gondii em búfalos (Bubalus bubalis) no Estado do Pará ${ }^{1}$
}

\author{
Jenevaldo Barbosa da Silva ${ }^{2 *}$, Adivaldo Henrique da Fonseca ${ }^{3}$, Stefano Juliano Tavares \\ de Andrade ${ }^{4}$, André Guimarães Maciel e Silva ${ }^{4}$, Carlos Magno Chaves Oliveira ${ }^{4}$ \\ e José Diomedes Barbosa ${ }^{4}$
}

\begin{abstract}
Silva J.B., Fonseca A.H., Andrade S.J.T., Silva A.G.M., Oliveira C.M.C. \& Barbosa J.D. 2013. [Serological prevalence of Toxoplasma gondii in water buffaloes (Bubalus bubalis) in Marajó Island, State of Pará, Brazil.] Prevalência de anticorpos anti-Toxoplasma gondii em búfalos (Bubalus bubalis) no Estado do Pará. Pesquisa Veterinária Brasileira 33(5):581-585. Laboratório de Imunodiagnóstico, Departamento de Patologia Veterinária, Facudade de Ciências Agrárias e Veterinárias, Universidade Estadual Paulista (Unesp), Via de Acesso Prof. Paulo Donato Castellane s/n, Jaboticabal, SP 14884-900, Brazil. E-mail: jenevaldo@hotmail.com

The aim was to study the seroprevalence of Toxoplasma gondii in water buffaloes (Bubalus bubalis) from State of Pará, Brazil. Three hundred and nineteen buffaloes were randomly selected into seven municipalities of Marajó Island. For comparative purposes, 128 buffaloes of five municipalities in the state of Pará were also evaluated. The seroprevalence of T. gondii was evaluated by Indirect Enzyme Linked Immunosorbent Assay (iELISA). The samples diagnosed as positive in iELISA were subjected to Immunofluorescence Antibody Test (IFAT). We evaluated risk factors: location, breed, pregnancy and co-infection with Brucella abortus or Mycobacterium bovis. The frequency of animals positive for T. gondii in iELISA were compared by chi-square $(\chi 2)$ with $95 \%$ confidence. Variables with $p<0.2$ were subjected to logistic regression analysis; the model was built based on the "odds ratios" test. The prevalence of $T$. gondii in iELISA was 41,6\% (186/447). In IFAT, 86,5\% (161/186) had their positivity for $T$. gondii confirmed. The average prevalence in the municipalities of the Marajó Island and of the mainland was 32\% (103/319) and 55\% (70/128), respectively. The municipalities with the highest prevalence were Soure (53\%) and Salvaterra (49\%) in Marajó Island, and Castanhal (55\%) and Thailândia (50\%) in the Continent. The breed and co-infection with Brucella abortus or Mycobacterium bovis presented no influence on the prevalence of T. gondii. Additionally, pregnant animals were $57 \%$ more positive for $T$. gondii than nonpregnant animals. The presence of antibodies is an indicative of T. gondii in buffaloes in the state of Pará, and these findings represent a risk not only for farm animals, but to public health as a source of infection.

INDEX TERMS: Water buffaloes, iELISA, IFAT, Toxoplasma gondii, Pará.
\end{abstract}

\footnotetext{
${ }^{1}$ Recebido em 30 de janeiro de 2013.

Aceito para publicação em 2 de março de 2013.

${ }^{2}$ Laboratório de Imunoparasitologia, Departamento de Patologia Veterinária, Facudade de Ciências Agrárias e Veterinárias (FCAV), Universidade Estadual Paulista (Unesp), Via de Acesso Prof. Paulo Donato Castellane s/n, Jaboticabal, SP 14884-900, Brasil. *Autor para correspondência: jenevaldo@hotmail.com

${ }^{3}$ Laboratório de Doenças Parasitárias, Departamento de Epidemiologia e Saúde Pública, Universidade Federal Rural de Rio de Janeiro (UFRRJ), BR 465 Km 7, Seropédica, RJ 23890-000, Brazil. E-mail: adivaldo@ufrrj.br

${ }^{4}$ Instituto de Medicina Veterinária, Universidade Federal do Pará, Rodovia BR 316 Km 61, Bairro Saudade, Centro Castanhal, PA 68740-970, Brasil. E-mail: Diomedes@ufpa.br
}

RESUMO.- O objetivo do estudo foi conhecer a prevalência sorológica de Toxoplasma gondii em búfalos (Bubalus bubalis) do Estado do Pará, Brasil. Foram selecionados randomicamente 319 bubalinos distribuídos em sete municípios da Ilha do Marajó. Para efeito comparativo também foram avaliados 128 bubalinos pertencentes a cinco municípios do Estado do Pará. A prevalência sorológica de Toxoplasma gondii foi avaliada pelo Ensaio de Imunoadsorção Enzimático Indireto (iELISA). As amostras diagnósticadas como positivas no iELISA foram submetidas a Reação de Imunofluorescência Indireta (RIFI). Foram avaliados os fatores de risco: localidade, raça, gestação, co-infecção por Brucella 
abortus e co-infecção por Mycobacterium bovis. As frequências de animais positivos no iELISA para T. gondii foram comparadas pelo teste de Qui-quadrado ( $\chi 2$ ) com $95 \%$ de confiabilidade. As variáveis com $\mathrm{p}<0,2$ foram submetidos à análise de regressão logística, sendo o modelo construído baseado no teste da "odds ratios". A prevalência de T. gondii observada no iELISA foi de 41,6\% (186/447). Na RIFI, $86,5 \%(161 / 186)$ das amostram positivas no iELISA tiveram sua positividade para T. gondii confirmada. A prevalência média nos municípios da Ilha do Marajo e do Continente foi de $32 \%(103 / 319)$ e 55\% (70/128), respectivamente. Os municípios que apresentaram as maiores prevalências foram Soure (53\%) e Salvaterra (49\%) na Ilha do Marajó e Castanhal (55\%) e Tailândia (50\%) no Continente. Os fatores de risco raça e co-infecção por Brucella abortus ou Mycobacterium bovis não influenciaram na prevalência de T. gondii. Além disso, animais gestantes foram $57 \%$ mais positivos para T. gondii do que animais não gestantes. A circulação de anticorpos é um indicativo da presença do agente da toxoplasmose em búfalos no Estado do Pará. Esses achados representam um risco não apenas para os animais de produção, mas à saúde pública, como uma fonte de infecção.

TERMOS DE INDEXAÇÃO: Bubalinos, iELISA, RIFI, Toxoplasma gondii, Pará

\section{INTRODUÇÃO}

A toxoplasmose é uma importante zoonose de caráter cosmopolita, possuindo uma elevada a prevalência em seres humanos (Dubey 1996). A infecção em bovinos normalmente não causa sintomatologia clínica, pois estes possuem uma elevada resistência natural ao parasita (Dubey \& Thulliez 1994). Pouco se sabe sobre a infecção e prevalência de anticorpos anti-T. gondii em búfalos (Bubalus bubalis), porém estudos indicam ser menor do que em bovinos (Dubey et al. 1998, Huong et al. 1998, Gondim et al. 1999, Fujii et al. 2001).

A carne de animais persistentimente infectados é uma das mais importantes fontes de toxoplasmose humana (Lunden \& Uggla 1992), sendo necessário investigar a prevalência da infecção por T. gondii nos animais de produção. No Brasil, estudos desmontraram a presença e importância do T. gondii, especialmente em pequenos ruminantes (Amaral et al. 1978, Chiari et al. 1987, Sella et al. 1994, Gondim et al. 1999), no entanto, algumas regiões, como o estado do Pará, que possui o maior rebanho de búfalos e o quinto maior rebanho de bovinos do Brasil, possuem poucas informações sobre a prevalência da infecção.

A importância da carne de búfalos como fonte de infecção para seres humanos torna-se mais relevante quando se trata da Ilha do Marajó. Nesta região com um dos menores Índices de Desenvolvimento Humano (IDH) do país e onde o búfalo é considerado a maior fonte de proteína de origem animal da população, a ingestão de carne não inspecionada pelo sistema de vigilância municipal e/ou estadual é facilitada.

A realização deste estudo foi motivada pelas condições socioeconômicas da população morajoara, pela importân- cia da bubalinocultura no estado do Pará e pela carência de estudos sobre a prevalência de Toxoplasma gondii na região. Neste sentido, o objetivo deste trabalho foi conhecer a frequência de anticorpos anti-T. gondii em búfalos criados no Estado do Pará, Brasil.

\section{MATERIAL E MÉTODOS}

Foi realizado um estudo de soroprevalência transversal em bubalinos entre os anos de 2009 e 2011. Doze municípios do Estado do Pará/Brasil foram selecionados por conveniência e facilidade de acesso às propriedades. Na Ilha do Marajó foram selecionados os municípios: Soure, Salvaterra, Muaná, Chaves, Ponta de Pedras, Cachoeira do Arari e Santa Cruz do Arari. No continente foram selecionados os municípios: Belém, Castanhal, Tailândia, Paragominas e Moju.

O cálculo do tamanho mínimo da amostra foi determinado pela seguinte fórmula do Centro Panamericano de Zoonoses (1997):

$$
\mathrm{N}=\mathrm{p} .(100-\mathrm{p}) \mathrm{Z}^{2} /(\mathrm{d} \cdot \mathrm{p} / 100)^{2}
$$

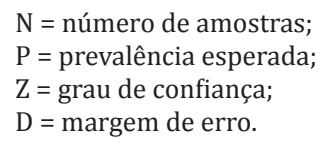

A prevalência esperada para anticorpos anti-Toxoplasma gondii em búfalos foi estimada em 50\%, baseando-se nos achados de Silva et al. (2010). 0 intervalo de confiança foi de 95\% e a margem de erro foi de $5 \%$. Deste modo, o tamanho mínimo da amostra foi de 361 animais.

Para o estudo, 447 animais fêmeas das raças Murrah e Mediterrânea foram selecionados, sendo 319 búfalas da Ilha do Marajó e 128 búfalas do Continente (Quadro 1).

Os títulos de anticorpos anti-Toxoplasma gondii foram determinados pelo Ensaio de Imunoadsorção Enzimático indireto (iELISA), conforme técnica descrita por Voller et al. (1976) e Reação de Imunofluorescência Indireta (RIFI), segundo Camargo (1964). Em ambas as técnicas, como controles positivos, foram utilizados soros de bubalinos que apresentaram altos título de anticorpos (iELISA e RIFI) e como controles negativos, utilizou-se soros de bubalinos recém-nascidos que não ingeriram colostro e reagiram negativamente no iELISA e RIFI.

Para o iELISA foi utilizado antígeno extraído de camundongos Swiss Webster (Mus musculus), infectados com taquizoítas da cepa

Quadro 1. Distribuição geográfica dos búfalos avaliados de acordo com a raça e o período reprodutivo, Estado do Pará, Brasil, 2009-2011

\begin{tabular}{lcccc}
\hline \multirow{2}{*}{ Municípios } & No de & \multicolumn{2}{c}{ Raças } & Gestante \\
\cline { 3 - 4 } & animais & Murrah & Mediterrâneo & \\
\hline Soure & 118 & 60 & 58 & 20 \\
Salvaterra & 39 & 22 & 17 & 10 \\
Muaná & 36 & 17 & 19 & 8 \\
Chaves & 18 & 9 & 9 & 5 \\
Ponta de Pedra & 18 & 10 & 8 & 5 \\
Cachoeira do Arari & 21 & 11 & 10 & 6 \\
Santa Cruz do Arari & 87 & 50 & 37 & 15 \\
Total Ilha do Marajó & 337 & 179 & 158 & 69 \\
Belém & 50 & 30 & 20 & 15 \\
Tailândia & 18 & 10 & 8 & 5 \\
Castanhal & 60 & 30 & 30 & 18 \\
Paragominas & 20 & 12 & 8 & 2 \\
Moju & 12 & 9 & 3 & 2 \\
Total Continente & 160 & 91 & 69 & 42
\end{tabular}


RH de T. gondii. A concentração ótima do antígeno para a sensibilização das placas foi de $5 \mu \mathrm{g} / \mathrm{mL}$. Os soros testes e controles positivos e negativos foram diluídos 1:200. A leitura foi realizada em leitor de iELISA, em um comprimento de onda de $405 \mathrm{~nm}$.

0 ponto de corte do teste foi determinado segundo Frey et al. (1998). Os soros de búfalos negativos foram utilizados para definir a linha de corte do ensaio, discernindo os animais positivos e negativos com nível de confiança de 99,0\%. A fórmula matemática de Frey et al. (1998) se baseia em um fator $t$ (distribuição t-Student), determinado pelo número de controles negativos e nível de confiança desejado:

$$
\text { Linha de corte }=X+S D \times t \sqrt{1}+(1 / n)
$$

$X=$ média das densidades ópticas dos controles negativos.

$S D=$ desvio padrão das densidades ópticas dos controles negativos.

$t=$ valor da distribuição $t$, baseado em $n$ e no de nível de confiança desejado.

$n=$ número de controles negativos.

Para corrigir o efeito da variação da densidade óptica (DO) obtida com a leitura de cada placa testada, o valor da linha de corte de cada uma das placas foi igualado a 100 (DO soro teste x 100/ linha de corte) e os resultados de cada soro teste expressos na forma de Índice de Densidade Óptica (IDO).

Para a RIFI foram utilizadas lâminas sensibilizadas com taquizoítas de T. gondii produzidos em camundongos Swiss Webster (Mus musculus), soros controles positivos e negativos e soro anti-bovino conjugado com isotiocianato de fluoresceína (F-7887TM, Sigma-Chemical, EUA). As amostras foram analisadas nas diluições de 1:64, 1:100, 1:200, 1:400 e 1:800. 0 ponto de corte foi de 1:64 segundo Garcia et al. (1999).

Os resultados sorológicos para T. gondii foram agrupados de acordo com os fatores de risco: municípios, localidades (Continente e Ilha), raça (Murrah e Mediterrâneo), co-infecção por Brucella abortus e por Mycobacterium bovis e período reprodutivo (gestante e não gestante).

O diagnóstico de brucelose e tuberculose foi realizado por veterinários habilitados pelo Ministério da Agricultura Pecuária e Abastecimento (MAPA), segundo normativa do Programa Nacional de Controle e Erradicação da Brucelose e da Tuberculose (PNCEBT) do Brasil (Brasil, 2006). As análises foram realizadas no Laboratório Nacional de Agropecuária (Lanagro). 0 diagnóstico de brucelose foi realizado em duas etapas. Inicialmente foi realizado o teste do antígeno acidificado a como triagem. Os animais que reagiram positivo ao teste de antígeno acidificado foram submetidos a um teste confirmatório, o 2-Mercaptoetanol, que é mais específico. Para tuberculose o teste cervical simples foi a prova de triagem e o teste cervical comparativo foi a prova confirmatória para animais reagentes.

As frequências de animais positivos no iELISA para T. gondii foram comparadas pelo teste de Qui-quadrado $\left(\chi^{2}\right)$ com $95 \%$ de confiabilidade. As variáveis com $\mathrm{p}<0,2$ foram submetidas à análise de regressão logística, sendo o modelo construído baseado no teste da "odds ratios". Os procedimentos operacionais foram realizados no software Rstudio, Foundation computação estatística, versão 2.12.2 (2011).

\section{RESULTADOS}

A prevalência de Toxoplasma gondii observada pelo iELISA e pela RIFI foi de 41,6\% (186/447) e 36,0\% (161/447), respectivamente. Portanto, 86,5\% (161/186) das amostram positivas no iELISA tiveram sua positividade para $T$. gondii confirmada na RIFI (Quadro 2). Os títulos de anticor- pos nos 161 búfalos positivas na RIFI foram: $64(62,71 \%)$, 100 (24,84\%), 200 (9,31\%) e 400 (3,10\%).

A prevalência média de T. gondii em búfalos na Ilha do Marajo e Continente foi de 32\% (103/319) e 55\% (70/128), respectivamente. Os municípios que apresentaram as maiores prevalênciasforam Soure (53\%) e Salvaterra

\begin{tabular}{|c|c|c|}
\hline \multicolumn{3}{|c|}{$\begin{array}{c}\text { Quadro 2. Prevalência sorológica de } \\
\text { Toxoplasma gondii por Ensaio de } \\
\text { Imunoadsorção Enzimático indireto } \\
\text { (iELISA) e Reação de Imunofluorescência } \\
\text { Indireta (RIFI) }\end{array}$} \\
\hline \multirow[t]{2}{*}{ T. gondii } & \multicolumn{2}{|c|}{ RIFI } \\
\hline & Positivo & Negativo \\
\hline \multicolumn{3}{|l|}{ ELISA } \\
\hline Positivo & 161 & 25 \\
\hline Negativo & 0 & 0 \\
\hline
\end{tabular}

Quadro 3. Prevalência média de búfalos soropositivos para Toxoplasma gondii em búfalos de sete municípios da Ilha do Marajó e cinco municípios do Continente, Estado do Pará, Brasil, 2009-2011

\begin{tabular}{lccccc}
\hline \multicolumn{1}{c}{ Fator de risco } & $\mathrm{N}$ & $\mathrm{P}(\%)$ & $\chi^{2}$ & $\mathrm{RP}$ & $p$-valor \\
\hline Ilha do Marajó & & & & & \\
$\quad$ Soure* & 118 & 53 & - & - & - \\
$\quad$ Salvaterra & 39 & 49 & 7,46 & 1,08 & 0,0630 \\
$\quad$ Muaná & 36 & 03 & 29,09 & 17,67 & 0,0001 \\
$\quad$ Chaves & 18 & 44 & 11,32 & 1,20 & 0,0545 \\
$\quad$ Ponta de Pedra & 16 & 06 & 12,54 & 8,83 & 0,0004 \\
Cachoeira do Arari & 18 & 27 & 4,09 & 1,96 & 0,0429 \\
$\quad$ Santa Cruz do Arari & 87 & 15 & 31,73 & 3,53 & 0,0001 \\
Continente & & & & & \\
$\quad$ Belém* & 50 & 46 & - & - & - \\
$\quad$ Tailândia & 18 & 50 & 0,08 & 0,92 & 0,7706 \\
$\quad$ Castanhal & 60 & 55 & 0,88 & 0,84 & 0,3471 \\
$\quad$ Paragominas & 20 & 15 & 10,10 & 3,07 & 0,0033 \\
$\quad$ Moju & 12 & 25 & 3,51 & 1,84 & 0,0063 \\
Localidades & & & & & - \\
$\quad$ Continente* & 128 & 55 & - & - & - \\
$\quad$ Ilha & 319 & 32 & 35,92 & 1,71 & 0,0001
\end{tabular}

* Valor referência, $\mathrm{N}=$ número de animais avaliados, $\mathrm{P}(\%)$ = Prevalência de soropositivitos para T. gondii, $\chi^{2}=$ qui-quadrado, $\mathrm{RP}=$ razão de prevalência, IC = intervalo de confiança.

Quadro 4. Prevalência média de búfalos soropositivos para Toxoplasma gondii de acordo com os fatores de risco raça, positividade para brucelose e tuberculose e período reprodutivo, Estado do Pará, Brasil, 2009-2011

\begin{tabular}{lccccc}
\hline \multicolumn{1}{c}{ Fator de risco } & $\mathrm{N}$ & $\mathrm{P}(\%)$ & $\chi^{2}$ & $\mathrm{RP}$ & $p$-valor \\
\hline Raça & & & & & \\
$\quad$ Mediterrâneo* & 227 & 30,2 & - & - & - \\
$\quad$ Murrah & 270 & 33,5 & 0,40 & 0,90 & 0,5240 \\
$\quad$ Brucelose & & & & & - \\
$\quad$ Positivo* & 15 & 33,3 & - & - & - \\
$\quad$ Negativo & 482 & 32,0 & 0,05 & 1,04 & 0,8202 \\
$\quad$ Tuberculose & & & & & \\
$\quad$ Positivo* & 20 & 40,0 & - & - & - \\
$\quad$ Negativo & 477 & 37,3 & 0,93 & 0,66 & 0,0335 \\
Gestação & & & & & \\
$\quad$ Positiva* & 111 & 39,0 & - & - & - \\
$\quad$ Negativa & 386 & 24,9 & 5,06 & 1,57 & 0,0244 \\
\end{tabular}

* Valor referência, $\mathrm{N}$ = número de animais avaliados, $\mathrm{P}(\%)$ = Prevalência de soropositivitos para T. gondii, $\chi^{2}=$ qui-quadrado, $\mathrm{RP}=$ razão de prevalência, IC = intervalo de confiança. 
(49\%) na Ilha do Marajó e Castanhal (55\%) e Tailândia (50\%) no Continente. No Continente, Paragominas e Mojú apresentaram uma prevalência significativamente inferior $(\mathrm{p}<0.05)$ aos demais municípios. Na Ilha, todos os municípios apresentaram prevalência para T. gondii significativamente inferior $(\mathrm{p}<0.05)$ a Soure e Salvaterra. Porém, atenção especial deve ser dada aos bubalinos dos municípios de Muaná (3\%) e Ponta de Pedra (6\%) que foram em sua maioria negativos (Quadro 3).

Os fatores de risco raça e co-infecção por Brucella abortus ou Mycobacterium bovis não influenciaram na prevalência de T. gondii em búfalos. Por outro lado, búfalas gestantes foram $57 \%$ mais positivos para T. gondii do que búfalas não gestantes (Quadro 4).

\section{DISCUSSÃO}

No Brasil poucos trabalhos foram realizados sobre ocorrência de anticorpos anti-Toxoplasma gondii em bubalinos. Alguns estudos realizados no Brasil demonstraram uma prevalência do agente em búfalos inferior ao observado em bovinos. Gondim et al. (1999) na Bahia, Fujii et al. (2001) em São Paulo e Barros et al. (1999) e Silva et al. (2010) no Pará observaram que 3,85\%, 3,2\%,12,2\% e 1,1\% dos búfalos foram sororreagentes, respectivamente. Essa baixa prevalência é similar às observadas em outros países (Dubey et al. 1998, Huong et al. 1998, Navidpour \& Hoghooghi-Rad 1998).

A alta prevalência de bubalinos sororreagentes a T. gondii observada neste estudo diverge da maioria dos achados nacionais e internacionais (Huong et al. 1998, Dubey et al. 1998, Gondim et al. 1999, Silva et al. 2010). Navidpour; Hoghooghi-Rad (1998) afirmaram que a infecção de búfalos por T. gondii é pouco comum. No entanto, Dubey \& Beattie (1988) na Turquia e Souza et al. (2001) no estado de São Paulo-Brasil, observaram $48,0 \%$ e $49,9 \%$ de bubalinos sororreagentes para $T$. gondii, respectivamente.

Essa discrepância de achados é um incentivo para novas pesquisas, pois a maioria dos estudos indica que a toxoplasmose é pouco disseminada entre bubalinos. Essa diferença nos resultados pode estar diretamente relacionada à sensibilidade e especificidade das técnicas de diagnóstico empregadas nos diferentes estudos e às amostragens utilizadas. A padronização de um ponto de corte para o iELISA ou mesmo a determinação da qualidade dos antígenos utilizados para a confecção das lâminas de RIFI devem ser uma preocupação eminente.

Na literatura são poucos os estudos realizados sobre prevalência de anticorpos anti- T. gondii em búfalos que levaram em consideração a relação de possíveis fatores de risco com a prevalência do parasito. Neste estudo, observamos que, além de uma diferença significativa $(\mathrm{p}<0,05)$ entre a soroprevalência entre os animais dos diversos municípios estudados, o período reprodutivo demonstrou ser importante na ocorrência de toxoplasmose. Porém, não foi possível saber se as búfalas em gestação eram mais velhas do que as não gestantes e a idade seja o real fator de risco e não o estado reprodutivo do animal. Trabalhos apontam para alta associação entre aumento da idade e a presença de anticorpos anti-T. gondii em diferentes espécies animais, uma vez que com a idade aumenta a chance de contato com o coccício (Gondim et al. 1999, Dubey 1986).

\section{CONCLUSÕES}

A presença de búfalos soropositivos é um indicativo da circulação de Toxoplasma gondii em animais no Estado do Pará. A alta prevalência de animais positivos para T. gondii merece uma atenção especial, visto que búfalos são mantidos em uma relação íntima com outras espécies domésticas e silvestres. Ênfase especial deve ser dada a possível circulação do agente na Ilha do Marajó, onde o consumo de carne de búfalos é comum e pode representar um risco à saúde pública devido à toxoplasmose.

Embora os estudos epidemiológicos sejam ainda pouco aplicados em rebanhos bubalinos, atualmente faz-se necessário levar em consideração diversos fatores de risco ao inferir um levantamento de prevalência das diversas parasitoses que acometem os bubalinos no Brasil. Diversas variáveis que já foram confirmadas como fatores predisponentes às doenças em bovinos ainda necessitam ser melhores estudados em bubalinos.

Agradecimentos.- Ao Conselho Nacional de Desenvolvimento Científico e Tecnológico (CNPq) pelo apoio financeiro. à Professora Rosangela Zacarias Machado (FCAV/UNESP) por ceder o antígeno utilizado.

\section{REFERÊNCIAS}

Amaral V., Santos S.M. \& Rebouças M.M. 1978. Sobre a prevalência de anticorpos antitoxoplasma em soros de caprinos e ovinos procedentes respectivamente, dos Estados da Bahia e Rio Grande do Sul. Biológico, São Paulo, 44:331-340.

Barros A.A.M., Molnár E., Carvalho M. \& Molnár L. 1999. IELISA e latex-aglutinação utilizados para detectar anticorpos anti-Toxoplasma gondii em diferentes animais domésticos. Anais 20 Congresso Brasileiro de Microbiologia, Sociedade Brasileira de Microbiologia, Salvador, BA, p.143.

Camargo M.E. 1964. Improved technique of indirect immunofluorescence for serological diagnosis of toxoplasmosis. Revta Inst. Med. Trop., São Paulo, 6:117-118.

Chiari C.A., Lima W.S., Lima J.D. \& Antunes C.M.F. 1987. Soro-epidemiologia da toxoplasmose caprina em Minas Gerais, Brasil. Arq. Bras. Med. Vet. Zootec. 39:587-609.

Dubey J.P. 1986. A review of toxoplasmosis in cattle. Vet. Parasitol. 22:177202.

Dubey J.P. 1994. Toxoplasmosis. J. Am. Vet. Med. Assoc. 205:1593-1598.

Dubey J.P., Romand S., Hilali M., Kwok O.C.H. \& Thulliez P. 1998. Seroprevalence of antibodies to Neospora caninum and Toxoplasma gondii in water buffaloes (Bubalus bubalis) from Egypt. Int. J. Parasitol. 28:527529.

Frey A. Canzio J.D. \& Zurakowski D. 1998. A statistically defined endpoint titer determination method for immunoassays. J. Immunol. Methods 221:35-41.

Fujii T.U., Kasai N., Vasconcellos S.A., Richtzenhain L.J., Cortez A., Souza S.L.P., Baruselli P.S., Nishi S.M., Ferreira F. \& Gennari S.M. 2001. Anticorpos anti-Neospora caninum e contra outros agentes de abortamentos em búfalas da Região do Vale do Ribeira, São Paulo, Brasil. Arqs Inst. Biológico, São Paulo, 66:5-9.

Huong L.T.T., Ljungstrom B.L.L., Uggla A. \& Bjorkman C. 1998. Prevalence of antidodies to Neospora caninum and Toxoplasma gondii in cattle and buffaloes in southern Vietnam. Vet. Parasitol. 75:53-57.

Garcia J.L., Navarro I.T., Ogawa L. \& Oliveira R.C. 1999. Soroprevalência do Toxoplasma gondii em suínos, bovinos, ovinos e eqüinos e sua correla- 
ção com humanos, felinos e caninos, oriundos de propriedades rurais do norte do Paraná, Brasil. Ciência Rural 29:91-97.

Gondim L.F.P., Barbosa Jr H.V., Ribeiro Filho C.H.A. \& Saeki H. 1999. Serological survey of antibodies to Toxoplasma gondii in goats, sheep, cattle and water buffaloes in Bahia State, Brazil. Vet. Parasitol. 82:273-276.

Lundén A. \& Uggla A. 1992. Infectivity of Toxoplasma gondii in mutton following curing, smoking, freezing or microwave cooking. Int. J. Food Microbiol. 15:357-363.

Navidpour S. \& Hoghooghi-Rad N. 1998. Seroprevalence of anti-Toxoplasma gondii antibodies in buffaloes in Khoozestan province, Iran. Vet. Parasitol. 77:191-194.

Sella M.Z., Navarro I.T., Vidotto O., Freire R.L. \& Shida P.N. 1994. Epidemiologia da toxoplasmose caprina: levantamento sorológico de Toxoplasma gondii em caprinos leiteiros na microrregião de Londrina, Paraná, Brasil. Braz. J. Vet. Parasitol. 3:13-16.

Silva S.P., Mota R.A., Faria E.B., Fernandes E.F.T.S., Neto O.L.S., Albuquerque P.P.F. \& Dias H.L.T. 2010. Anticorpos IgG anti-Neospora caninum e Toxoplasma gondii em búfalas (Bubalus bubalis) criadas no estado do Pará. Pesq. Vet. Bras. 30:443-446.

Souza L.M., Nascimento A.A., Furuta P.I., Basso L.M.S., Silveira D.M. \& Costa A.J. 2001. Detecção de anticorpos contra Neospora caninum e Toxoplasma gondii em soros de bubalinos (Bubalus bubalis) no Estado de São Paulo, Brasil. Ciênc. Agrárias 22:39-48.

Voller A., Bidwell D.E. \& Bartlett A. 1976. Enzyme immunoassays in diagnostic medicine. Bull. World Health Organization, Genève, 53: 55-65. 